\title{
Perturbation Monte-Carlo Based Derivative Estimation for Imaging Low-Scattering Inclusions in a Heterogeneous Tissue
}

\author{
Y. Phaneendra Kumar and R. M. Vasu \\ Department of Instrumentation, Indian Institute of Science \\ Bangalore - 560012 , INDIA \\ vasu@isu.iisc.ernet.in
}

\begin{abstract}
For near infrared imaging of neonatal head surfounded by cerebrospinal fluid layer, we have developed an imaging modality, which uses perturbation Monte-Carlo estimation of Jacobian of the forward model for photon propagation. In this case, we have shown that the conventional techniques, which uses diffusion approximation for light transport in tissue, fails to give the reconstruction of tissue optical properties. We were able to successfully reconstruct both scattering- and absorption inhomogeneities in objects which fall under the transport regime. Results of numerical simulation are presented.
\end{abstract}

\section{INTRODUCTION}

Near-infrared spectroscopy (NIRS) of the neonatal brain for monitoring change in oxygenation level has caught good attention [1][2] among the research groups working on biomedical imaging. Out of different layers of neonatal head, namely scalp and skull, cerebrospinal fluid (CSF), and gray and white matter, the low-scattering CSF layer influences, significantly, the path taken by photons and even the depth of penetration [3][4].

The analytic models for the light transport in tissue use diffusion equation.(DE) that is an approximation to radiative transfer equation (RTE). Diffusion approximation fails in voidlike regions where the scattering coefficient $\left(\mu_{s}\right)$ is small [4] (i.e. regions where scattering-mean-free-path is large). RTE distinguishes between the scattering- and absorbing objects inside media of such nature through use of directional information of the photons. Inversion methods based on Monte-Carlo simulation, which solves RTE, should be able to reconstruct the heterogeneous tissue properties in these cases. There were many attempts [5][6] to solve the inverse photon transport problem using $\mathrm{MC}$ simulations.

The major drawback of a stochastic method, like the MC simulation, is large computation time involved in taking many millions of photons through the tissue. Inversion that makes use of repeated application of such forward model would become too expensive to be a useful option. This issue has been addressed in [6] making use of perturbation
Monte-Carlo (pMC) methods to extract Frechet derivative information required to solve the inverse problem. The $\mathrm{pMC}$ requires only single $\mathrm{MC}$ 'simulation, with the derivative extraction rapidly done using available analytical formulae for derivatives. In reference [6], this was used to solve a simple two region inverse problem of photon migration in a heterogeneous slab.

In this work, we extend the pMC approach to construct the full Jacobian matrix, which is used in a non-linear optimization algorithm (locally linearized) to reconstruct both absorption- and scattering heterogeneities. We show that the inversion algorithm using Jacobian obtained from this MC-based method is able to give superior reconstruction when the diffusion equation is no longer valid, i.e., when the scattering coefficient $\left(\mu_{s}\right)$ is small and comparable to absorption coefficient $\left(\mu_{\mathrm{a}}\right)$.

This paper is organized as follows. Section-2, briefly introduces the MC simulation for propagation of light through tissue and describe the pMC-based derivative estimation. Section-3 has the details of simulations and the non-linear optimization algorithm, which uses the derivative information, for reconstruction of optical properties. The results of reconstruction of optical inhomogeneities in lowscattering media are also presented in Section-3. Finally we give the concluding remarks and outline our future work.

\section{Monte-Carlo (MC) SIMUlaton AND}

PERTURBation MONTE-CARLo. (PMC) BASED DERIVATIVE ESTIMATION

\section{Monte-Carlo (MC) Simulation}

Light transport through turbid media can be described stochastically wherein absorption and scattering of photons are individually tracked, resulting in photon histories. A large number of photons are launched into the tissue and traced through probabilistically defined absorption and scattering until they either exit the tissue or are absorbed. 


\section{TENCON $2003 / 530$}

For statistically meaningful results, a large number, usually 6-10 million, of photons are required to be launched. The measurements at the detector are usually the photon arrival time, pathlength distribution and temporal point spread function (TPSF). Using three uniformly distributed random numbers $x_{1}, x_{2}$, and $\dot{x}_{3}$, in eq (1); we calculate the distance travelled between two successive scattering events, $s$, the angles of scattering, one azimuthal $(\psi)$ and one polar $(\phi)$. The total attenuation coefficient, $\mu_{\mathrm{t}}$, is defined as, $\mu_{\mathrm{t}}=\mu_{\mathrm{a}}+\mu_{\mathrm{s}}$. The function $\mathrm{f}(\psi)$ in eq. (1) is known as the cumulative probability phase function [7]. For every interaction of photon in the tissue, it looses $\left(\mu_{\mathrm{a}} / \mu_{4}\right)$ of its incident weight and scatters with new weight $\left(\mu_{s} / \mu_{\mathrm{t}}\right)$ times the old weight. Total pathlength traveled by photon is calculated by summing all the individual scattering lengths. The time of travel is obtained by dividing this total path length by velocity of light in the tissue.

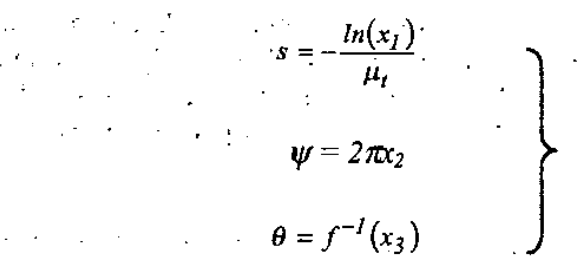

The MC simulation, being a stochastic process, has the drawback of using large computation time to arrive at histograms of photons. To overcome this limitation, a hybrid Monte-Carlo was developed which makes use of only one full $\mathrm{MC}$ simulation [5].

\section{Perturbation Monte-Carlo (pMC) in the Inversion problems}

Currently available reconstruction schemes in optical tomography reconstructs perturbations in $\mu_{a}$ and $\mu_{s}$, with prior knowledge of the background optical properties; $\mu_{\mathrm{a}}{ }^{b}$ and $\mu_{s}^{b}$. The simplified perturbation equation [8], solved to get back the update vector for optical properties, namely $\left[\Delta \mu_{a}, \Delta \mu_{s}\right]$, is

$$
\Delta M \cong F^{\prime}\left[\mu_{a^{b}}{ }^{b}, \mu_{s}{ }^{b}\right]\left[\Delta \mu_{a}, \Delta \mu_{s}^{\prime}\right]
$$

where, $\Delta M$ is the change in measurement owing to perturbations in $\mu_{\mathrm{a}}$ and $\mu_{\mathrm{s}} . F$ is the forward model used for light transport through the medium and $F^{\prime}$ is its Frechet derivative, also referred to as the Jacobian. DE-based methods are available [9] to quickly estimate Jacobian. However,'such derivatives obtained from the $\mathrm{DE}$ model which is valid only when, $\mu_{\mathrm{a}}<\dot{<} \mu_{\mathrm{s}}$ are erroneous, when $\mu_{\mathrm{s}}$ is small and comparable to $\mu_{\mathrm{a}}$. In such cases, MC-based methods help us to construct more accurate derivative information

- Perturbation Monte-Carlo (pMC) is used to quickly get the derivative information. We use this information in a nonlinear optimization algorithm to get the reconstruction of optical properties of the tissue. The perturbation in $\mu_{\mathrm{a}}{ }^{\mathrm{b}}$ and $\mu_{\mathrm{s}}^{\mathrm{b}}$ are introduced in specific locations using the stored formation of distribution of $\mu_{\mathrm{d}}^{b}$ and $\mu_{\mathrm{s}}^{\mathrm{b}}$. The new photon weight due to these perturbations in $\mu_{\mathrm{a}}$ and $\mu_{\mathrm{s}}$ is evaluated using the analytic expression (eq. (3)) used in neutron transport [6][10,11]. We can get the modified time histograms due to these perturbations in $\mu_{\mathrm{s}}$ and $\mu_{\mathrm{s}}$ in much less computational time than one full $\mathrm{MC}$ simulation for the background medium.

If $\delta \mu_{\mathrm{a}}$ and $\delta \mu_{\mathrm{s}}$ are the perturbations in certain locations in $\mu_{\mathrm{s}}$ and $\mu_{\mathrm{s}}$ respectively, the absorption- and scattering coefficients become, $\bar{\mu}_{a}=\mu_{a}+\delta \mu_{a}$ and $\bar{\mu}_{s}=\mu_{s}+\delta \mu_{s}$ then the photon weight $w$ changes to $\bar{w}$ as

$$
\bar{w}=w\left(\frac{\bar{\mu}_{s} / \mu_{t}}{\mu_{s} / \mu_{t}}\right)^{n}\left(\frac{\bar{\mu}_{t}}{\mu_{t}}\right)^{n} \exp \left[-\left(\bar{\mu}_{t}-\mu_{t}\right)\right]
$$

where, $n$ is the total number of collisions the photon undergoes in the perturbed region and $l$ is the total pathlength of the photon in the perturbed region. Eq. (3) provides a way to estimate changes in measured photon density (or weight) owing to changes in $\mu_{\mathrm{a}}$ and $\mu_{\mathrm{s}}$ in specific localities. We use the analytical expression given in eq. (3) to calculate the Jacobian matrices $\left\{\left(\partial w / \partial \delta \mu_{\mathrm{a}}\right) \&\left(\partial w / \partial \delta \mu_{s}\right)\right\}$. The Jacobian matrix, which has as many rows as there are source-detector pairs, has rows built up of the derivative terms using eq. (3), one each for each node. The values of $n$ $\& l$ for the derivative calculation are pre-calculated and stored for each sub-region, which covers $\approx 5$ grid elements surrounding each element. Strategy for calculation of the derivative is listed below in Fig. (1). For $\mu_{\mathrm{a}}$ derivative $n \& l$ do not change, but for, $\mu_{s}$-derivative these are re-estimated as $\mu_{s}$ changes the number of scattering events in the perturbed region. But, since the Jacobian is a sparse matrix, with non-zero elements only around the source and detector, we use a priori information of geometry for quick calculation of $n \& l$ in only these relevant regions. This reduces the computation time greatly.

for each source-detector pair

for all the nodes, $\mathrm{i}=1-\mathrm{N}$ ascertain $l$ and $n$ from the initial MC simulation calculate $\left(\partial w / \partial \delta \mu_{\mathrm{a}}\right)$ through Eq. (2) calculate $\left(\partial w / \partial \delta \mu_{s}\right)$ through Eq. $(2)^{\dagger}$ end

end

${ }^{\dagger}-$ For $\mu_{s}$ derivative, new estimate of $l$ and $n$ are obtained only for those regions where non-zero contribution to the Jacobian is expected.

Figure 1. Strategy for calculation of Jacobian 


\section{Simulations AND RESUlts}

\section{Medical. Image Processing /531}

\section{Details of Simulation}

We have considered a tissue-mimicking phantom, cylindrical in shape, of diameter $66 \mathrm{~mm}$. The object was divided into $67 \times 67$ grids of uniform size and the optical properties are considered constant in each grid element. The centre of the main cylinder is chosen to be $(34,34)$. The inhomogeneity is also cylindrical in shape, with diameter 12 mm centered at $(34,50)$. The anisotropy factor, $g$, was kept at 0.9 . We have considered the following two cases of inhomogeneity (See Fig. 3): (a) The background optical properties are $\mu_{\mathrm{a}}{ }^{\mathrm{b}}=0.040 \mathrm{~mm}^{-1} \& \mu_{\mathrm{s}}^{\mathrm{b}}=0.5 \mathrm{~mm}^{-1}$ and the inhomogeneity optical properties are $\mu_{a}^{\text {in }}=0.14 \mathrm{~mm}^{-1}$ with $\mu_{\mathrm{s}}^{\text {in }}$ same as $\mu_{\mathrm{s}}^{\mathrm{b}}$; (b) $\mu_{\mathrm{a}}^{\text {in }}$ same as $\mu_{\mathrm{a}}^{\mathrm{b}}=0.040 \mathrm{~mm}^{-1}$ and $\mu_{\mathrm{s}}^{\text {in }}$ $=5 \mathrm{~mm}^{-1} \& \mu_{\mathrm{s}}^{\mathrm{b}}=10 \mathrm{~mm}^{-1}$ (i.e. $\mu_{\mathrm{s}}^{\text {in }}<\mu_{\mathrm{s}}^{\mathrm{b}}$ ).

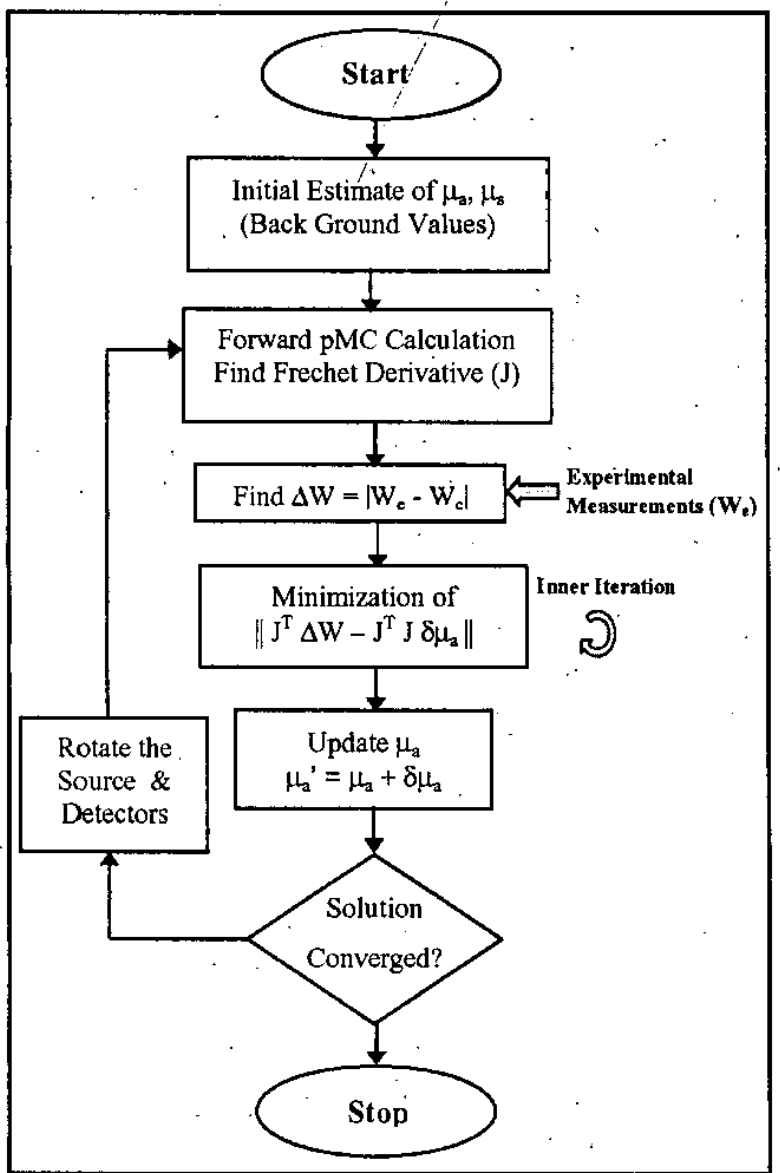

Figure 2. The flowchart used in iterative reconstruction. The inner loop outputs optimal parameter values used by the outer loop to update the optical coefficients.

We have simulated the forward projection data, which was integrated intensity, through $\mathrm{MC}$ simulation. A $2 \%$ Gaussian noise was added to the temporal point spread function

before integration. For each source, data are gathered at 11 equally spaced detectors kept on the opposite side (see Fig. 2). The simulation is repeated for 11 equally spaced locations of the source for each of which the. relative detector locations are unchanged.

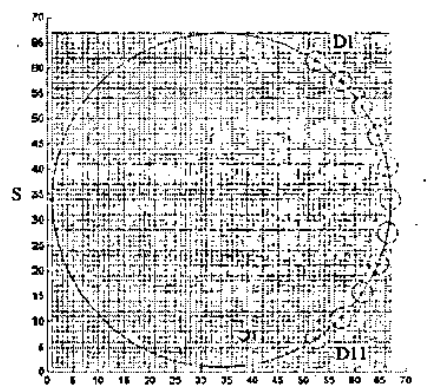

Figure 3. The data gathering geometry for simulation. The source is at S, and D1-D11 are detectors

(a)

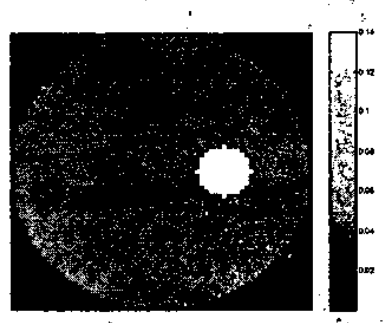

(b)

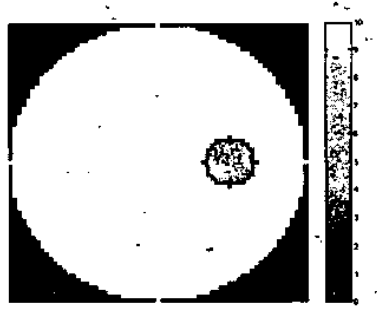

Figure 4. Original object distributions; (a) Background $\mu_{\mathrm{a}} \&$ $\mu_{s}$ are $0.04 \mathrm{~mm}^{-1} \& 0.5 \mathrm{~mm}^{-1}$ respectively, and the inclusion has $\mu_{\mathrm{a}}=0.14 \mathrm{~mm}^{-1} \& \mu_{\mathrm{s}}=0.5 \mathrm{~mm}^{-1}$. (b) Background $\mu_{\mathrm{a}}=0.04 \mathrm{~mm}^{-1} \& \mu_{\mathrm{s}}=10 \mathrm{~mm}^{-1}$ and the inclusion has $\mu_{\mathrm{a}}=0.04 \mathrm{~mm}^{-1} \& \mu_{\mathrm{s}}=5 \mathrm{~mm}^{-1}$.

For each iteration, one source -11 detector combination (i.e. one view in tomography terminology) provides the complete data set. This is similar to the approach of reference [12] of using a 'propagation - backpropagation' strategy wherein we solve the perturbation equation to get the update for optical parameters from each view, before considering the next view in the subsequent iteration. One set of iterations is said to be completed, when we run through data from all the sources.

The Jacobian matrix is computed using the method described in Section-2 and the algorithm described in Fig. 2 is used to extract the update vectors, which are added to the initial guess to arrive at the object distributions. 


\section{TENCON $2003 / 532$}

\section{Results}

The algorithm converged in 9 and 16 iterations respectively for the two different types of objects considered. The convergence criterion used was that the norm of the difference between computed data and the experimental data should be below a, preset, small value. Since a stochastic procedure like the MC gives a lot of spike noise in the data, the reconstruction required smoothing using averaging filters.

Fig.4 shows the cross-section of the original objects (the two cases). The reconstructions in the two cases are shown in Fig. 5, where perturbation Monte-Carlo (pMC) method is used to construct the Jacobian. For absorption inhomogeneity above the background level, the inhomogeneity location and reconstruction are found reasonably close to the original values.

(a)

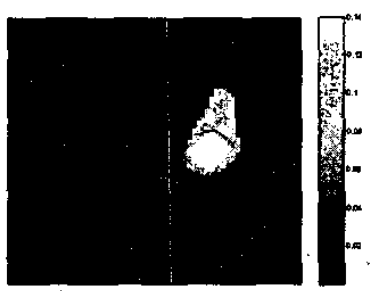

(b)

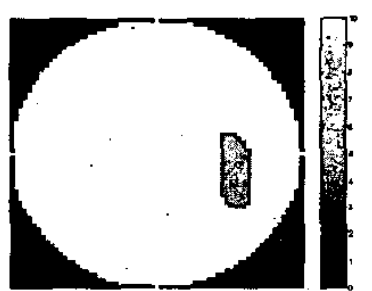

Figure 5. Reconstruction obtained from pMC-based algorithm. (a) \& (b) are objects shown Fig. 4(a) \& (b) respectively. The values of the optical coefficients at the centre of the inhomogeneities are $0.139 \mathrm{~mm}^{-1} \& 4.873 \mathrm{~mm}^{-1}$ respectively for the two cases.

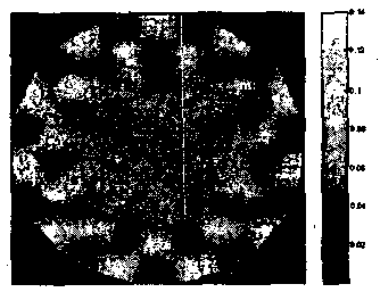

Figure 6. For low-scattering coefficient background. reconstruction from a standard algorithm using the diffusion equation.

The results presented required smoothing through a local averaging filter. The result for the absorption inhomogeneity (Fig. 5(a)) can be compared to the result from the TOAST algorithm [13] (Fig. 6), which makes use of the DE for light propagation. Here, because of low scattering cross-section, the DE is not valid and consequently the TOAST algorithm fails to locate the object.

For the second case, where the inhomogeneity is in scattering coefficient, $\mu_{s}$, which is lower than the background (Fig. 4(b)), the reconstruction had many false clusters around the actual inhomogeneity. The result presented in Fig. 5(b) is after eliminating these clusters through a special image-processing algorithm, which selectively suppresses clusters [14]. After this postprocessing the result in Fig. 5(b) is able to locate the scattering inhomogeneity. In this case TOAST algorithm did notconverge to a solution.

\section{Conclusions}

We have developed an imaging modality for reconstructing the optical inhomogeneities in a low scattering tissue. We have shown that, the conventional techniques, which use DE fail in these cases. We use a pMC-based method for estimation of Jacobian. The non-linear optimization algorithm, which uses this derivative information for reconstruction of optical properties of tissue reconstructed both $\mu_{\mathrm{a}}$ - and $\mu_{\mathrm{s}}$ inhornogeneities from low-scattering media. We can use this method to image the neonatal head cerebral spinal fluid layer, which is almost non-scattering compared to the gray- and white-matter in the head. This approach can even image the clear synovial fluid in joints, which plays an important role in the optical detection of rheumatoid arthritis.

Even though we can use the analytic form of the perturbation equation for simultaneous reconstruction of $\mu_{\mathrm{a}}$ and $\mu_{3}$ inhomogeneities, we considered each case separately. Results of simultaneous $\mu_{\mathrm{a}}$ and $\mu_{\mathrm{s}}$, which would conclusively prove the effectiveness of the pMC-based algorithm will be presented in a future work.

\section{ACKNOWLEDGEMENTS}

The Authors would like to thank.Mr. S. Chandra Sekhar, $\mathrm{ECE}$, for the useful discussions on the clustering algorithms.

\section{REFERENCES}

[1] J. E. Brazy, D. V. Lewis, M.H. Mitnick, and F. F. Jobsis, "Noninvasive monitoring of cerebral oxygenation in preterm infants: Preliminary observations," Pediatrics 25, 217-225, 1985.

[2] J. S. Wyatt, D. T. Delpy, M. Cope, S. Wray, and E.O.R. Reynolds, "Quantification of cerebral oxygenation and haemodynamics in sick newborn infants by near infrared spectroscopy," Lancet ii, 1063-1066, 1986. 
[3] E. Okada, M. Firbank, M. Schweiger, S. R. Arridge, M. Cope, and D. T. Delpy, "Theoretical and experimental investigation of near infrared light propagation in a model of the adult head," Appl. Opt. 36, 21-31, 1997.

[4] M. Firbank, S. R. Arridge, M. Schweiger, and D. T. Delpy, "An investigation of light transport through scattering bodies with non-scattering regions," Phys. Med. Biol. 41, 767-783, 1996.

[5] A. Sassaroli, C. Blumeti, F. Martelli, L. Alianelli, D. Contini, A. Ismaelli, and G. Zaccanti,. "Monte Carlo procedure for investigating light propagation and imaging of highly scattering media," Appl. Opt. 37, 7392-7400, 1998.

[6] C. K. Hayakawa, J. Spanier, F. Bevilacqua, A.K. Dunn, J.S. You, B.J. Tromberg, and V. Venugopalan, "Perturbation Monte Carlo methods to solve inverse photon migration problems in heterogeneous tissues," Opt. Lett. 26, 1335-1337, 2001.

[7] Y. Yamada, "Light-tissue interaction and optical imaging in biomedicine," Annual Review of Heat Transfer 6, Ed. C.-L. Tien, Begell House, New York, Chap. 1, 1-50, 1995.

[8] A. H. Hielscher, A. D. Klose, and K. M. Hanson, "Gradient-based iterative image reconstruction scheme for time-resolved optical tomography," IEEE Trans. Med. Imag. 18, 262-271, 1999.

[9] S. R. Arridge, and M. Schweiger, "A gradient-based optimisation scheme for optical tomography," Optics Express 6, 213-226, 1998.

[10] J. Spanier, and E. M. Gelbard, Monte Carlo Principles and Neutron Transport Problems, Addison-Wesley, Reading, Mass., 1969.

[11] H. Rief, "Generalized Monte Carlo perturbation algorithms for correlated sampling and a second-order Taylor series approach," Ann. Nucl. Energy 9, 455-476, 1984.

[12] F. Natterer, and F. Wübbeling, "A propagationbackpropagation method for ultrasound tomography," Inverse Problems 11, 1225-1232, 1995.

[13] S. R. Arridge, and M. Schweiger, "The use of multiple data types in Time-resolved Optical Absorption and Scattering Tomography (TOAST)," Proc. SPIE 2035, 218-229, 1993.,

[14] R. M. Haralick, S. R. Sternberg, and X. Zhuang, "Image analysis using mathematical morphology," IEEE Trans. Patt. Anal. Mach. Intel. 9(4), 532-560, 1987. 